\title{
Impact on Caregivers of Patients with Myeloproliferative Neoplasms (MPNs) in the United States: Results from the Living with MPNs Survey
}

\author{
${ }^{1}$ Incyte Corporation, Wilmington, DE, USA \\ ${ }^{2}$ University of Colorado Anschutz Medical Campus, Aurora, CO, USA \\ ${ }^{3}$ San Antonio Cancer Center - An NCI Designated Cancer Center, San Antonio, TX, USA
}

Dilan Paranagama ${ }^{1 *}$, Shreekant Parasuraman ${ }^{1}$, Jingbo $\mathrm{Yu}^{1}$, Lindsey Lyle $^{2}$ and Ruben Mesa ${ }^{3}$

*Corresponding author: Dilan Paranagama, PhD, Incyte Corporation, 1801 Augustine Cut-Off, Wilmington, DE 19803, USA, Tel: +1-302-498-5780, Fax: +1-302-425-2789

\begin{abstract}
Background: To describe the impact of myeloproliferative neoplasms (MPNs) on caregivers.

Methods: 904 patients with MPNs aged 18-70 years in the US who completed the online Living with MPNs survey between April and November, 2016. The MPN Symptom Assessment Form Total Symptom Score was used to describe symptom burden. Patient-reported need for caregiver assistance and caregivers' employment change due to MPNs was reported.

Results: $55.1 \%$ of patients reported receiving caregiver assistance. Caregivers were predominantly family members (spouses/partners [68.1\%], sons/daughters [10.6\%], parents [6.2\%]) and most commonly provided assistance with homemaking $(77.7 \%)$, companionship $(57.4 \%)$, and transportation $(55.2 \%)$. Most caregivers were employed (76.9\%), of whom $26.3 \%$ reduced work hours, $7.4 \%$ terminated a job, and $6.6 \%$ retired early to care for a patient with an MPN. Patients who received caregiver assistance reported a higher mean MPN-SAF total symptom score (40.9 vs. 20.0) and had more comorbid conditions (52.4\% vs. $38.4 \%)$ than patients not receiving caregiver assistance.

Conclusions: Most survey respondents to this US survey reported receiving MPN-related caregiver assistance. A higher symptom burden was reported by those receiving assistance than those who did not. Approximately onefourth of caregivers underwent employment adjustments. There may be a need for support services for patients with MPNs and their caregivers.
\end{abstract}

\section{Keywords}

Myeloproliferative neoplasm, Polycythemia vera, Myelofibrosis, Essential thrombocythemia, Caregiver, Employment

\begin{abstract}
Abbreviations
COPD: Chronic Obstructive Pulmonary Disease; ET: Essential Thrombocythemia; MF: Myelofibrosis; MPN: Myeloproliferative Neoplasm; MPN-SAF: MPN Symptom Assessment Form; PV: Polycythemia Vera; TSS: Total Symptom Score
\end{abstract}

\section{Introduction}

Patients with a myeloproliferative neoplasm (MPN), including myelofibrosis (MF), polycythemia vera (PV), and essential thrombocythemia (ET) [1], have reduced survival compared with the general population [2] and experience a variety of burdensome symptoms $[3,4]$. Annual incidence rates for ET, PV, and PMF are estimated at 1.03, 0.84, and 0.47 per 100,000, respectively [5]. Common symptoms include fatigue, inactivity, concentration problems, bone pain, night sweats, early satiety, and difficulty sleeping, with some patients reporting that their symptoms were "very severe" [4]. Overall, MPN-related symptoms have a prominent influence on patient quality of life (QoL) [4] and may increase the need for caregiver assistance [6]. Additionally, treatment schedules and the frequency of clinic follow-up are heterogeneous and may require varying degrees of caregiver involvement.

Cancer often places significant burdens on family members who help care for patients in need [7]. These burdens may be subjective, such as emotional 
toll [8] and changes to self-esteem [9], or objective, such as the number of hours of care provided, physical responsibilities associated with providing care (eg, added housework), and economic strains (eg, due to a reduced ability of the caregiver to work) [10-13]. Furthermore, the degree of caregiver burden can be affected by the patient's disease characteristics and caregiver disposition, as modelled by the adapted bio-behavioral Pittsburgh Mind Body Center Model [14]. The effects of caregiving on work productivity include increased absenteeism and an impaired ability to complete certain work-related tasks (known as presenteeism) $[10,12]$, with an estimated 40 million adults in the United States (US) providing unpaid care for another adult [15]. One study of patients diagnosed with various cancers, including primarily colon, lung, or breast cancer, showed that caregiver burden was positively correlated with deteriorating emotional functioning and increasing dyspnea in the patients under their care [16], supporting a direct influence of patient health and QoL on the burden experienced by their caregivers. These findings raise the possibility that more effective disease management may positively affect caregivers by reducing patient disease burden and, thus, alleviating the associated caregiver stress.

Similar to other cancers, patients with MPNs often require assistance from a caregiver, with approximately $40 \%$ of patients reported receiving caregiver services in the international MPN Landmark survey [6]. However, limited data are available regarding the specific burden on caregivers attending to patients with MPNs and how healthcare providers may be able to help alleviate the burden. The goal of the present analysis was to evaluate the impact of MPNs on caregivers as reported by patients with MPNs in the US, including the type of assistance required, amount of time spent providing assistance, effect of these activities on caregivers' employment, and the association between disease severity and level of caregiving needed.

\section{Methods}

\section{Patients and study design}

The Living with MPNs survey was cross-sectional and conducted online in English between April and November 2016 to evaluate the impact of MPNs on employment and everyday life among patients in the US [17]. Patients 18 to 70 -years-old with a diagnosis of MF, PV, or ET were eligible to participate in the survey.

Respondents were recruited to the survey through emails and/or postcards sent by MPN groups; posts on patient advocacy websites; posts on MPN-focused social media (eg, patient group Facebook pages); text or banner ads through Google, Facebook, and selected medical websites; and postcards sent to oncologist and hematologist offices for distribution (a convenience sample). The study received approval from the Quorum
Review Institutional Review Board (Quorum Review File number 31458) and all survey respondents provided informed consent electronically.

\section{Assessments}

The Living with MPNs survey contained approximately 100 questions including the respondent's demographics (eg, age and sex); MPN diagnosis and disease-related medical history; changes in patient employment status; MPN-related symptoms; QoL measures (ie, functional measures); need for assistance with activities of daily living (ie, caregiver status); and impact on caregivers. Co-morbidities were reported by patients by choosing from a pre-populated list, and respondents could select as many as applicable ('Other' was an option). The impact of MPNs on caregivers was addressed with a series of survey questions within the Living with MPNs survey. The survey defined caregiver as "the person who assists you with your activities of daily living". Respondents chose answers from pre-populated lists to categorize the caregiver-respondent relationship (spouse/partner, son/daughter, parent, sibling, home healthcare professional, other relative, other) and type of help received, entered the amount of time spent receiving assistance in the past 7 days (range $0-720$ hours), and categorized the effect of these activities on caregivers' employment through responses to a series of yes/no statements. All survey responses were provided exclusively by patients.

The Living with MPNs survey included the MPN Symptom assessment form (MPN-SAF), a validated cancer distress screening tool used to assess symptom severity for 10 symptoms (abdominal discomfort, bone pain, early satiety, fatigue, fever, inactivity, itching, night sweats, problems with concentration, and unintentional weight loss). Individual symptom severity scores were graded on a scale from 0 (absent) to 10 (worst imaginable) and were used to calculate a total symptom score (TSS; sum of 10 individual symptom severity scores) [3]. Symptoms were further categorized by severity based on MPN-SAF score (none, 0; mild, 1-3; moderate, 4-6; severe, $\geq 7$ ).

In addition, impairments in physical, mental, and social functioning were assessed within the Living with MPNs survey with 15 questions based on the following Likert-like response options: "not at all", "a little bit", "somewhat", "quite a bit", and "a great deal".

\section{Statistical analyses}

Survey respondents were stratified by whether they did versus did not require caregiver assistance. Survey data were analyzed using descriptive statistics. Continuous variables were summarized with mean, standard deviation, median, minimum, and maximum, and categorical variables were summarized with number and percentage within each group. All analyses were conducted using SAS 9.4 (Cary, North Carolina). 
Table 1: Demographics and clinical characteristics of respondents by caregiver assistance.

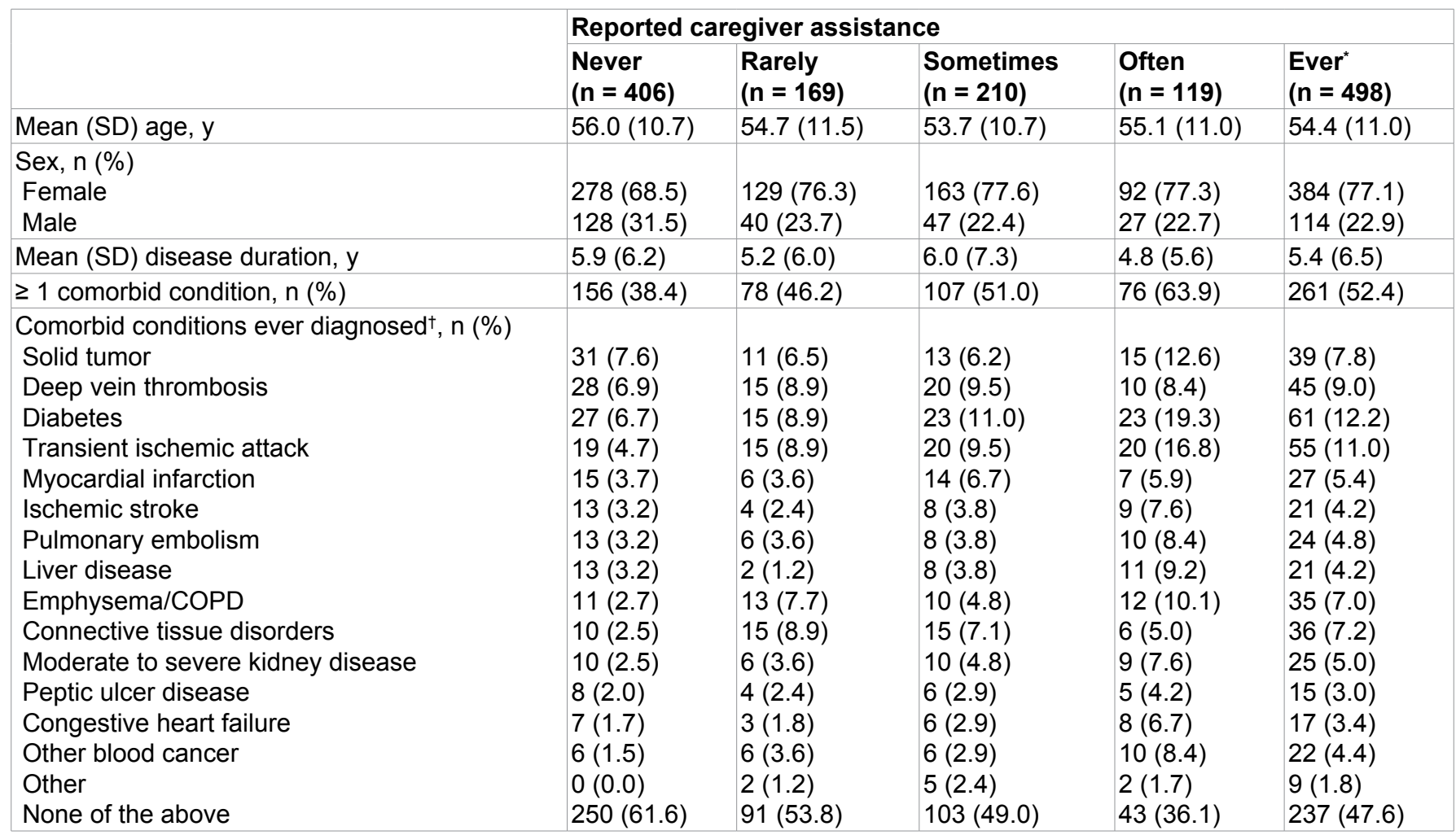

COPD: Chronic Obstructive Pulmonary Disease; "Includes all respondents who reported receiving help from a caregiver "rarely", "sometimes", or "often"; †Self-reported and based on a prepopulated list of select conditions. Respondents could select > 1 condition.

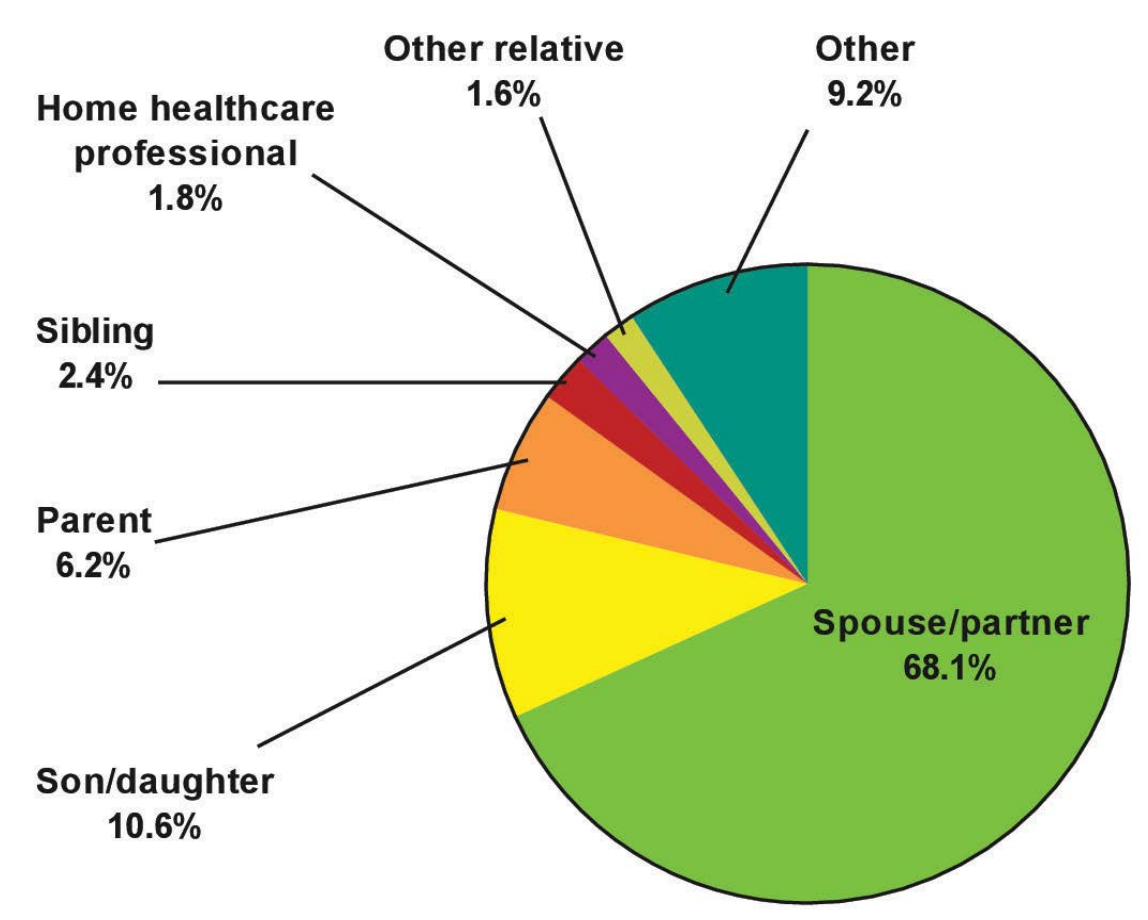

Figure 1: Caregiver. Respondents were patients who ever reported having a caregiver $(n=498)$. Caregiver options were selected from a list provided in the survey (spouse/partner, son/daughter, parent, sibling, home healthcare professional, other relative, other).

\section{Results}

\section{Respondent demographics and clinical}

\section{characteristics}

The survey was completed by 904 respondents with MPNs, of whom 498 (55.1\%) reported ever receiving help from a caregiver because of MPNs (term chosen by the respondent: rarely, $n=169$; sometimes, $n=210$; often, $\mathrm{n}=119$ ). Mean age was approximately 55 years, and the majority of respondents were female (77.1\%). Mean disease duration was similar between respondents who never had a caregiver versus those who did (5.9 vs. 5.4 
years). Compared with respondents who never required a caregiver, respondents who ever had a caregiver were more likely to have been diagnosed with one or more comorbid conditions (38.4\% vs. $52.4 \%$; Table 1 ).

\section{Caregiver assistance and employment}

The 3 most common types of caregivers were spouse/ partner $(68.1 \%)$, son/daughter $(10.6 \%)$, and parent (6.2\%; Figure 1). Assistance provided by caregivers most often included homemaking $(77.7 \%)$, companionship (57.4\%), and transportation (55.2\%; Table 2). Among all respondents, the mean (SD) amount of caregiver time required in the previous 7 days was 14.1 (22.3) hours. Of patients with a caregiver, $33.9 \%$ reported rarely receiving caregiver assistance (mean [SD] total hours in past 7 days, 6.6 [12.7] hours), $42.1 \%$ reported sometimes receiving assistance (10.8 [11.4] hours), and $23.9 \%$ reported often receiving assistance (30.4 [35.8] hours).
Most respondents indicated that their caregiver was employed at the time of their MPN diagnosis $(n=376$; 76.9\%). Among employed caregivers, $26.3 \%$ reduced work hours, $7.4 \%$ terminated a job, and $6.6 \%$ took early retirement to care for a person with an MPN (Figure 2).

\section{Respondent symptom burden}

Mean (SD) MPN-SAF TSS was lower among respondents who never had a caregiver compared with those who ever did (20.0 [17.1] vs. 40.9 [19.7], respectively) with a corresponding increase in caregiver assistance (rarely, 33.7 [17.7]; sometimes, 40.2 [19.2]; often, 52.4 [18.2]). Similarly, greater individual symptom severity was associated with an increasing respondent need for caregiver assistance (Figure 3). Fatigue or tiredness was the most frequently reported symptom and the symptom most commonly reported as "severe", regardless of the level of caregiver assistance.

Table 2: Patient-reported types of caregiver assistance due to MPNs.

\begin{tabular}{|c|c|c|c|c|}
\hline \multirow[t]{2}{*}{ Respondents, n (\%) } & \multicolumn{4}{|c|}{ Reported caregiver assistance } \\
\hline & $\begin{array}{l}\text { Rarely } \\
(n=169)\end{array}$ & $\begin{array}{l}\text { Sometimes } \\
(n=210)\end{array}$ & $\begin{array}{l}\text { Often } \\
(n=119)\end{array}$ & $\begin{array}{l}\text { Ever }^{*} \\
(n=498)\end{array}$ \\
\hline \multicolumn{5}{|c|}{ Type of caregiver assistance $^{\dagger}$} \\
\hline Homemaking & $99(58.6)$ & $173(82.4)$ & $115(96.6)$ & $387(77.7)$ \\
\hline Companionship & $86(50.9)$ & $121(57.6)$ & $79(66.4)$ & $286(57.4)$ \\
\hline Transportation & $72(42.6)$ & $119(56.7)$ & $84(70.6)$ & $275(55.2)$ \\
\hline Managing finances & $38(22.5)$ & $64(30.5)$ & $56(47.1)$ & $158(31.7)$ \\
\hline Healthcare assistance & $15(8.9)$ & $39(18.6)$ & $32(26.9)$ & $86(17.3)$ \\
\hline Personal care assistance & $3(1.8)$ & $9(4.3)$ & $23(19.3)$ & $35(7.0)$ \\
\hline
\end{tabular}

"Includes all respondents who reported receiving help from a caregiver "rarely", "sometimes", or "often"; tRespondents may have given $>1$ response.

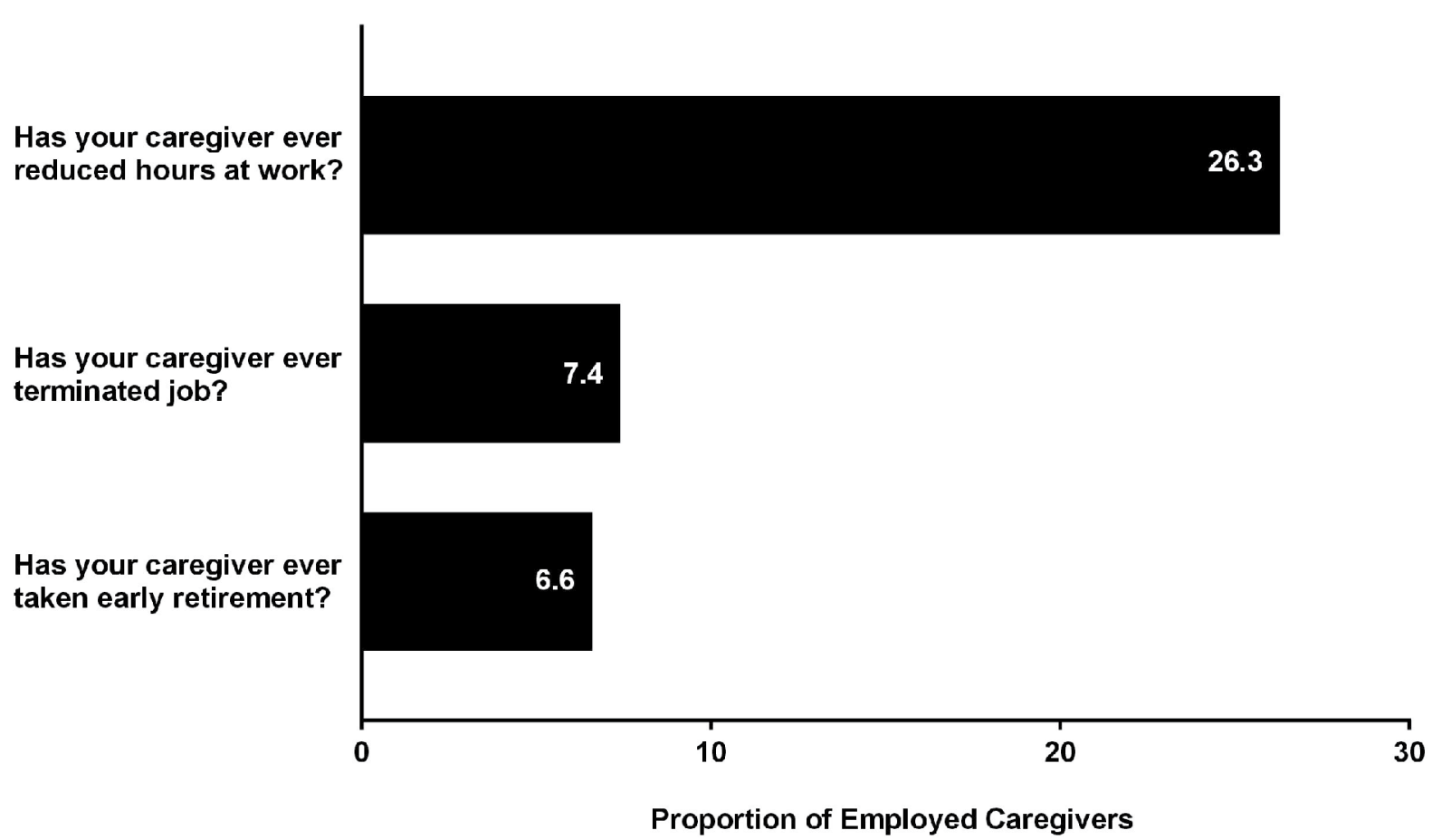

Figure 2: Employment changes of caregivers due to patients' MPNs. Respondents were patients who ever reported having a caregiver. Respondents may have given > 1 response. Percentages represent patients who responded "yes" to questions about employed caregivers $(n=376)$. 


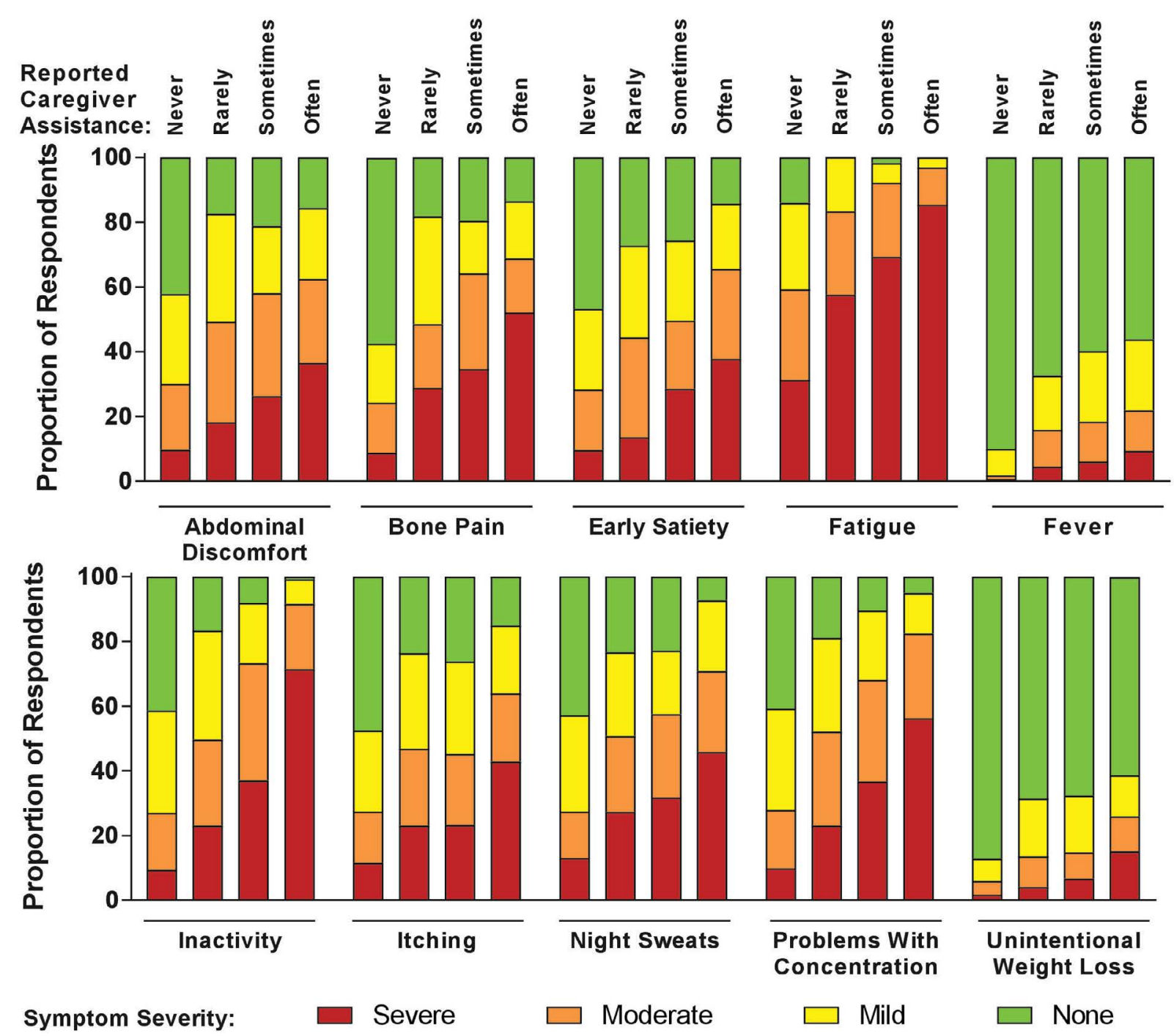

Figure 3: Respondent symptom severity by caregiver need. Percentages represent patient responses to questions about their symptom severity (chosen from the following options: severe, moderate, mild, or none). Symptom severity scores were compared between subgroups defined by reported level of caregiver assistance needed (chosen from the following options: never, $n=406$; rarely, $n=169$; sometimes, $n=210$; often, $n=119$ ).

\section{Functional impairments}

Increasing respondent need for caregiver assistance was generally associated with greater respondent functional impairments in all measures assessed by the survey. Of the 15 total functional measures assessed, the need to rest more often was the most common impairment, regardless of the level of caregiver assistance (Figure 4).

\section{Discussions}

The burden of cancer extends beyond patients and affects family members who provide caregiver services $[7,12]$. In the present survey of 904 respondents with MPNs, 55.1\% had received assistance from a caregiver, in agreement with findings from the MPN Landmark survey [6]. Caregivers were predominantly family members (ie, spouses/partners, sons/daughters, or parents), and their caregiving role commonly included homemaking, companionship, and transportation.

A goal of this study was to measure the patient-per- ceived burden on caregivers of individuals with MPNs in the US. The major impact identified was change in caregiver employment, which has implications for financial well-being and time management. Of the $76.9 \%$ of caregivers who were employed at the time of the respondent's diagnosis in the present analysis, $26.3 \%$ reduced work hours, $7.4 \%$ had terminated a job, and $6.6 \%$ had taken early retirement to care for their patient with an MPN. Previous studies examining the roles of caregivers in cancer settings have described a reduced ability of caregivers to work and effects on caregivers' QoL [10-12,18]. A study of 146 employed caregivers of patients with advanced ovarian cancer reported the additive effects of absenteeism and presenteeism due to caregiving have a mean percentage of overall work productivity loss of approximately $21 \%$ [10]. The major contributors to impaired work productivity were the time and financial burdens associated with providing treatment, the time spent visiting providers, and the time spent performing patient-related household activities $[11,12]$, which is similar to the services caregivers of patients with an MPN were reported to provide in the 

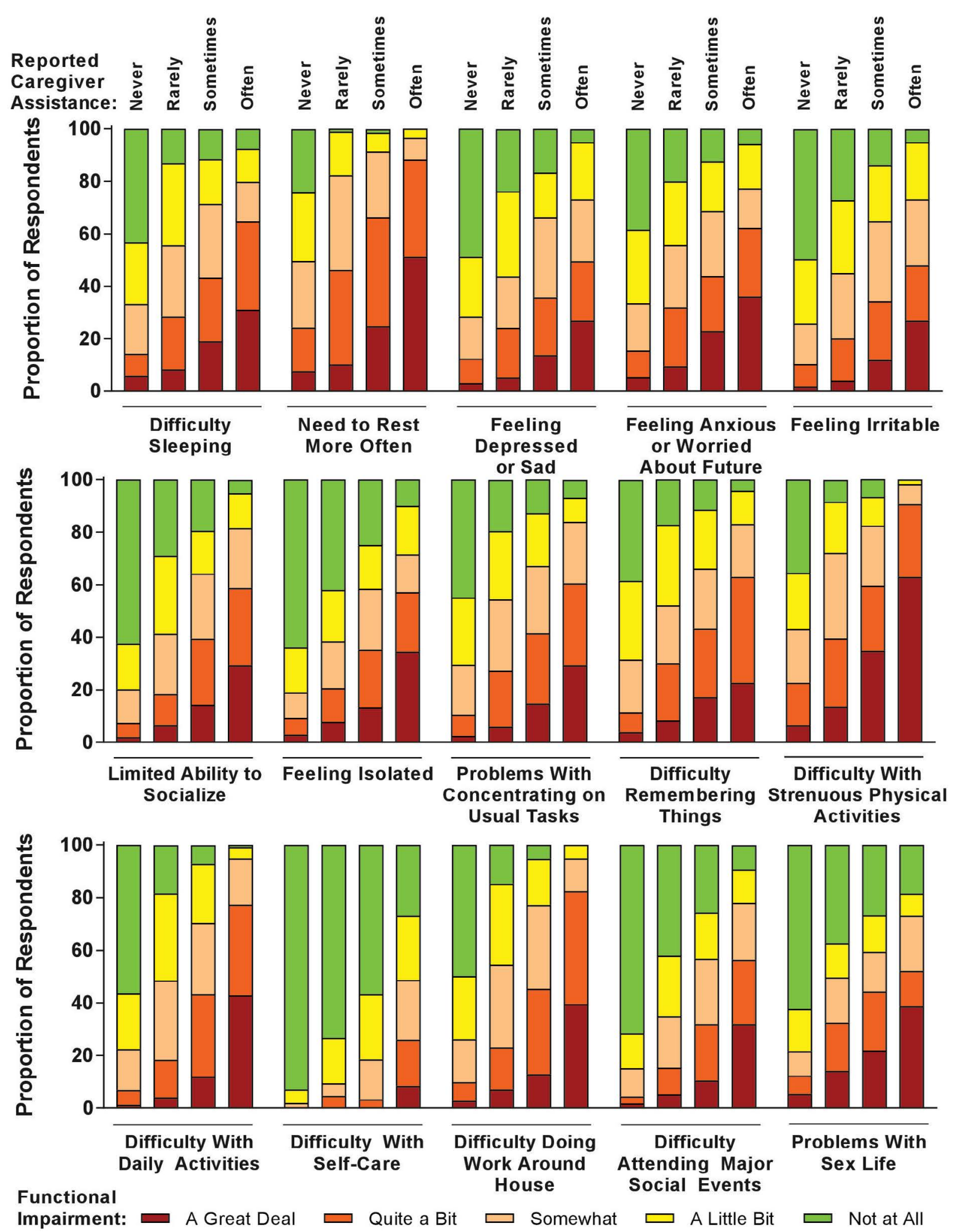

Figure 4: Respondent functional status by caregiver need. QoL, quality of life. Percentages represent patient responses to questions about how functional impairments affected QoL (chosen from the following options: A great deal, quite a bit, somewhat, a little bit, not at all). Effects of functional impairments on QoL were compared between subgroups defined by reported level of caregiver assistance (chosen from the following options: never, $n=406$; rarely, $n=169$; sometimes, $n=210$; often, $n=119)$.

current study. In a recent survey of 517 partners of women with nonmetastatic breast cancer, partners with higher perceived financial burdens had significantly worse mean QoL scores. While the majority of partners noted that it was important to maintain employment in order to remain insured, $32 \%$ required reduced work hours in order to care for their partner [18].
This study demonstrates a substantial burden on caregivers who provide assistance to a patient with an MPN, and caregivers are more often needed by patients with high MPN-related disease burden. This relationship between disease severity and care dependency has been reported by patients with a number of other diseases, as well [19-22]. Thus, the need for a caregiver 
may be reduced with improved symptom and disease management. MPN-related disease burden was generally greater among respondents who received caregiver assistance, including higher incidences of comorbid conditions at any time before the survey, more frequent and severe symptoms per the MPNSAF, and greater functional impairments at the time of survey completion.

\section{Implications for nursing}

The results from this analysis highlight an emerging need to develop management strategies for proactive symptom management by nurses and other healthcare professionals. Fatigue was found to be the most common and severe symptom for patients reported in the Living with MPNs survey and is often underreported as are other MPN symptoms [4]. Nurses should encourage patients to bring their caregivers to office visits as part of a holistic assessment of the impact of MPNs. Nurses are well-positioned to evaluate the total impact of the disease by interacting with both the patient and caregiver. Honea, et al. have provided a comprehensive review of nursing assessment and interventions to support caregivers in oncology. Briefly, they report education, support, psychotherapy, and respite interventions have the greatest effect in reducing caregiver strain and burden [23].

Findings from the Living with MPNs survey stress the importance of including the financial burden experienced by caregivers into the cost of cancer management. As a result of caring for a patient with an MPN, approximately one fourth of caregivers required employment adjustments that reduced their time spent working. Nurses involved in the care of patients with MPNs have the potential to play a unique role in mitigating caregiver burdens by providing information on resources such as professional caregiving services, transportation alternatives, the Family and Medical Leave Act [24], and facilitating access to state, local, and national support services, which can help relieve the financial and time burdens experienced by caregivers.

An unanswered question is whether improving MPN patient symptom and disease burden will lessen the need for, and impact on, caregivers; this question may be addressed with a prospective nurse-led study. In addition, increased nursing research into systematic assessment of patient and caregiver needs in order to provide tailored support would benefit both patient and caregiver.

\section{Limitations}

As with any online survey, there is a possibility of selection bias, and survey respondents may not be representative of the overall population of patients with MPNs. This analysis based on a US population may not be generalizable to other geographic regions considering differences in health care systems and insurance models. Patients were recruited via online advertising and targeted communications, and the survey was conducted online and in English; these factors may have favored recruitment of higher socioeconomic and better-educated individuals. Finally, all caregiver data were provided by patients rather than the caregivers themselves, and as such may under- or overestimate the true extent of caregiver burden.

\section{Conclusions}

Although results should not be generalized beyond the study population, most respondents reported receiving assistance from a caregiver, typically a family member, because of their disease. Based on patient reports, a significant proportion of caregivers required employment adjustments (eg, reduced work hours, terminated job, early retirement) to care for their patient with an MPN, potentially leading to personal and economic hardships. Nurses and other healthcare professionals involved in the care of patients with MPNs are uniquely positioned to help ameliorate the financial, functional, and societal burdens that patients and their caregivers often face by providing valuable information and by facilitating access to available support services.

\section{Acknowledgments}

DP, SP, and JY are employees and stockholders of Incyte Corporation. LL has served as a consultant for Celgene, Novartis, Incyte Corporation, Takeda Oncology, and Pfizer. RM received research funding from Incyte Corporation, Gilead, CTI, Promedior, and Celgene and served as a consultant for Ariad, Galena, and Novartis.

We thank Health Research Associates, Inc. for managing the web survey. Editorial assistance was provided by Tania Iqbal, PhD (Complete Healthcare Communications, LLC, New Wales, PA; a CHC Group company), whose work was funded by Incyte Corporation.

\section{Funding}

This work was funded by Incyte Corporation for all authors.

\section{References}

1. Arber DA, Orazi A, Hasserjian R, Thiele J, Borowitz MJ, et al. (2016) The 2016 revision to the World Health Organization classification of myeloid neoplasms and acute leukemia. Blood 127: 2391-2405.

2. Price GL, Davis KL, Karve S, Pohl G, Walgren RA (2014) Survival patterns in United States (US) medicare enrollees with non-CML myeloproliferative neoplasms (MPN). PLoS One 9: e90299.

3. Emanuel RM, Dueck AC, Geyer HL, Kiladjian JJ, Slot S, et al. (2012) Myeloproliferative neoplasm (MPN) symptom assessment form total symptom score: Prospective international assessment of an abbreviated symptom burden scoring system among patients with MPNs. J Clin Oncol 30: 4098-4103.

4. Mesa R, Miller CB, Thyne M, Mangan J, Goldberger $S$, et al. (2016) Myeloproliferative neoplasms (MPNs) 
have a significant impact on patients' overall health and productivity: The MPN Landmark survey. BMC Cancer 16: 167.

5. Titmarsh GJ, Duncombe AS, McMullin MF, O'Rorke M, Mesa R, et al. (2014) How common are myeloproliferative neoplasms? A systematic review and meta-analysis. Am J Hematol 89: 581-587.

6. Harrison CN, Koschmieder S, Foltz L, Guglielmelli P, Flindt T, et al. (2017) The impact of myeloproliferative neoplasms (MPNs) on patient quality of life and productivity: Results from the international MPN Landmark survey. Ann Hematol 96: 1653-1665.

7. Jeon SH, Pohl RV (2017) Health and work in the family: Evidence from spouses' cancer diagnoses. J Health Econ 52: 1-18.

8. Ullrich A, Ascherfeld L, Marx G, Bokemeyer C, Bergelt C, et al. (2017) Quality of life, psychological burden, needs, and satisfaction during specialized inpatient palliative care in family caregivers of advanced cancer patients. BMC Palliat Care 16: 31 .

9. Kim D (2017) Relationships between caregiving stress, depression, and self-esteem in family caregivers of adults with a disability. Occup Ther Int 2017: 1686143.

10. Angioli R, Capriglione S, Aloisi A, Miranda A, de Cicco Nardone C, et al. (2015) Economic impact among family caregivers of patients with advanced ovarian cancer. Int $\mathrm{J}$ Gynecol Cancer 25: 1541-1546.

11. Hartnett J, Thom B, Kline N (2016) Caregiver burden in end-stage ovarian cancer. Clin J Oncol Nurs 20: 169-173.

12. Kamal KM, Covvey JR, Dashputre A, Ghosh S, Shah S, et al. (2017) A systematic review of the effect of cancer treatment on work productivity of patients and caregivers. J Manag Care Spec Pharm 23: 136-162.

13. Bastawrous M (2013) Caregiver burden--a critical discussion. Int J Nurs Stud 50: 431-441.

14. Sherwood PR, Given BA, Donovan H, Baum A, Given CW, et al. (2008) Guiding research in family care: A new approach to oncology caregiving. Psychooncology 17: 986-996.
15. National Alliance for Caregiving, AARP Public Policy Institute (2015) Caregiving in the U.S. 2015 Report.

16. Krug K, Miksch A, Peters-Klimm F, Engeser P, Szecsenyi J (2016) Correlation between patient quality of life in palliative care and burden of their family caregivers: $A$ prospective observational cohort study. BMC Palliat Care 15: 4.

17. Yu J, Parasuraman S, Paranagama D, Naim A, Dubinski D (2016) Impact of myeloproliferative neoplasms on patients' employment status and work productivity in the United States: Results from the living with MPN patient survey. Blood (ASH Annual Meeting Abstracts) 128.

18. Veenstra CM, Wallner LP, Jagsi R, Abrahamse $P$, Griggs $\mathrm{JJ}$, et al. (2017) Long-term economic and employment outcomes among partners of women with early-stage breast cancer. J Oncol Pract 13: e916-e926.

19. Montgomery W, Goren A, Kahle-Wrobleski K, Nakamura T, Ueda K (2018) Detection, diagnosis, and treatment of Alzheimer's disease dementia stratified by severity as reported by caregivers in Japan. Neuropsychiatr Dis Treat 14: 1843-1854.

20. Janssen DJ, Franssen FM, Wouters EF, Schols JM, Spruit MA (2011) Impaired health status and care dependency in patients with advanced COPD or chronic heart failure. Qual Life Res 20: 1679-1688.

21. Janssen DJ, Wouters EF, Schols JM, Spruit MA (2013) Care dependency independently predicts two-year survival in outpatients with advanced chronic organ failure. J Am Med Dir Assoc 14: 194-198.

22. Riedel O, Dodel R, Deuschl G, Klotsche J, Forstl H, et al. (2012) Depression and care-dependency in Parkinson's disease: Results from a nationwide study of 1449 outpatients. Parkinsonism Relat Disord 18: 598-601.

23. Honea NJ, Brintnall R, Given B, Sherwood P, Colao DB, et al. (2008) Putting evidence into practice: Nursing assessment and interventions to reduce family caregiver strain and burden. Clin J Oncol Nurs 12: 507-516.

24. US Department of Labor Family and Medical Leave Act. In: US Department of Labor, Wage and Hour Division. 